\title{
Propagación in vitro de Carica papaya var. PTM-331 a partir de meristemos apicales
}

\author{
In vitro propagation of Carica papaya var. PTM-331 from apical meristem
}

\author{
Reynaldo Solis L. ${ }^{1 *}$, Julio Olivera S. ${ }^{2}$ y Rafael S. La Rosa L. ${ }^{1}$
}

1 Universidad Nacional Federico Villarreal - Facultad de Ciencias Naturales y Matemática. Jr. Rio Chepen $\mathrm{N}^{\circ} 110,114$ y $290-\mathrm{El}$ Agustino.

*Email Reynaldo Solis Leyva: rsolisleyva@yahoo.com.pe

2 Estación Experimental Agraria DONOSO -Instituto Nacional de Innovación Agraria. Altura Km. 5,4 Innovación Agraria. Altura Km. 5,4
de la Carretera Chancay - Huaral. Aceptado: $\quad$ 15/12/2011 Publicado online: $08 / 02 / 2012$

\section{Resumen}

El trabajo consistió en desarrollar un protocolo de propagación in vitro de la variedad de papaya PTM-331 a partir de meristemos apicales, con la finalidad de obtener plántulas vigorosas y libres de enfermedades, empleando la técnica del cultivo de tejidos. Las yemas apicales empleadas fueron obtenidas de plantas cultivadas en invernadero, los cuales fueron usados como explantes para la extracción de meristemos. La mejor diferenciación de meristemos se logró en el medio basal MS suplementado con $0,5 \mathrm{mg} \cdot \mathrm{L}^{-1}$ de BAP, $0,5 \mathrm{mg} \cdot \mathrm{L}^{-1}$ de AIA y $10 \mathrm{mg} \cdot \mathrm{L}^{-1}$ de adenina. La mejor multiplicación se logró con el medio MS suplementado con $0,5 \mathrm{mg} \cdot \mathrm{L}^{-1}$ de BAP, 0,5 mg. $\mathrm{L}^{-1}$ de AIA y $0,3 \mathrm{mg} \cdot \mathrm{L}^{-1}$ de $\mathrm{AG}_{3}$, con un coeficiente de multiplicación de 3,42; mientras que el mejor medio para el enraizamiento fue la combinación del medio $\mathrm{MS}, 3 \mathrm{mg} \cdot \mathrm{L}^{-1}$ de AIB y $5 \mathrm{mg} \cdot \mathrm{L}^{-1}$ de adenina, donde se obtuvo $83,33 \%$ de plantas enraizadas.

Palabras claves: Carica papaya, Propagación in vitro, meristemos apicales, invernadero, explantes.

\section{Abstract}

An in vitro protocol was develop to propagate variety of papaya PTM-331 from apical meristems, with the objective of obtaining vigorous and disease-free seedlings, using tissue culture techniques. Apical buds were obtained from seedlings cultivated in greenhouse and used as explants for meristem dissection. Meristems were cultured on MS basal medium supplemented with $0,5 \mathrm{mg} \cdot \mathrm{L}^{-1}$ of BAP, $0,5 \mathrm{mg} \cdot \mathrm{L}^{-1}$ of AIA and $10 \mathrm{mg} \cdot \mathrm{L}^{-1} \mathrm{of}$ adenine for their differentiation. The best multiplication of explants was achieved with the combination of MS medium supplemented with $0,5 \mathrm{mg} \cdot \mathrm{L}^{-1}$ of BAP, $0,5 \mathrm{mg} \cdot \mathrm{L}^{-1}$ of AIA and $0,3 \mathrm{mg} \cdot \mathrm{L}^{-1}$ of $\mathrm{AG}_{3}$, where largest seedlings, with more shoots were obtained. The best medium for rooting was the combination of MS, $3 \mathrm{mg}^{-\mathrm{L}^{-1}}$ of AIB and $5 \mathrm{mg} \cdot \mathrm{L}^{-1}$ of adenine, where $83,33 \%$ of rooted plants were obtained.

Keywords: Carica papaya, in vitro propagation, apical meristem, greenhouse, explants.

\section{Introducción}

En el Perú, la papaya (Carica papaya L.) es una especie importante en la economía del poblador amazónico debido a su fruta de alto rendimiento y valor nutritivo. Su cultivo presenta una serie de ventajas como calidad de su fruto, desarrollo vegetativo corto y la cosecha semanal luego de haber iniciado la producción, permitiendo el rápido retorno del capital invertido. Según Carbajal y Remuzgo (2007), en los últimos diez años la Amazonía Peruana se ha constituido en el principal productor y abastecedor de papaya al mercado de Lima e involucra a más del $35 \%$ de los agricultores en las distintas etapas de la cadena productiva.

El Instituto de Investigación de la Amazonía Peruana (IIAP) viene trabajando en el programa de mejoramiento genético de la papaya, teniendo como objetivos principales mejorar la calidad del fruto, incrementar el rendimiento y desarrollar variedades tolerantes a enfermedades limitativas al cultivo (Carbajal \& Remuzgo 2007); así se ha logrado obtener la variedad PTM-331.

Existen diversas formas de propagar la papaya, sin embargo, desde el punto de vista comercial, el uso de semilla sexual es el más generalizado (Franciosi 1992) a pesar de las dificultades que se presentan al obtenerse plantas de diferente sexo y que a veces las plantas resultantes no reproducen exactamente las características de la planta originaria (Ibar 1979). La propagación vegetativa por medio de estacas o injertos no brindan los efectos deseados, las primeras son de lento desarrollo y las segundas degeneran y no mantienen las características (Posada 2005).

Una forma de obtener plantas libres de patógenos es a través del cultivo in vitro de meristemos que se fundamenta en el hecho de que la distribución de los microorganismos (virus, bacterias y micoplasmas) en los tejidos de la planta no es uniforme y su concentración tiende a disminuir progresivamente hacia el ápice del tallo (Jiménez 1998). Con el estudio de los procesos que ocurren en el cultivo de tejidos de la papaya se han desarrollado técnicas de micropropagación por organogénesis (Alvarado 1992, Baca 2002, Gallardo et al. 2002) y embriogénesis somática (Jimenez 1999, Ascencio et al. 2008) pero en ninguno de estos casos se trabajó con la variedad PTM-331.

El desarrollo de una metodología de micropropagación a partir de meristemos apicales es importante para conocer el comportamiento in vitro de esta variedad y apoyar la multiplicación de plantas élite de sexo femenino seleccionadas en campo por sus características fenotípicas especiales; además se puede realizar una cuidadosa selección del material genético, manejar grandes volúmenes de plántulas en espacios reducidos y mantener un stock de plantas libres de enfermedades. Este trabajo tuvo como objetivo determinar los requerimientos nutricionales y hormonales de Carica papaya var. PTM-331 en los medios de cultivo durante las fases de establecimiento de meristemos apicales, multiplicación y enraizamiento de las plántulas. 


\section{Materiales y métodos}

El presente trabajo se desarrolló en el Laboratorio de Biotecnología de la Estación Experimental DONOSO del Instituto Nacional de Innovación Agraria del Perú. Los meristemos fueron extraídos de yemas apicales obtenidas de plantas de 45 días provenientes de invernadero. Para la desinfección, los explantes fueron lavados con abundante agua y jabón y luego fueron transportados a la cámara de flujo laminar en donde fueron sumergidos en alcohol $70 \%$ durante 30 segundos y enjuagados tres veces con agua destilada estéril, luego fueron sumergidos en hipoclorito de sodio $1 \%$ durante 10 minutos y enjuagados tres veces con agua destilada estéril.

Fase de establecimiento de meristemos.- Luego de desinfectar las yemas apicales, con la ayuda de un microscopio estereoscopio se realizó la disección de meristemos. Los explantes vegetales se colocaron sobre una placa Petri (148 x $120 \mathrm{~mm}$.), se sujetaron con pinzas y con ayuda del bisturí se eliminaron los primordios foliares hasta dejar el meristemo descubierto. Después se realizó un corte en la base del meristemo y se introdujo al tubo de ensayo que contenía el medio de cultivo.
Con la finalidad de obtener el medio de cultivo adecuado que nos permita la diferenciación de los meristemos se formularon diversos tratamientos considerando los resultados obtenidos por Alvarado (1992) y Baca (2002), empleando el medio de cultivo basal Murashige y Skoog (MS-1962) con diferentes concentraciones de BAP, ANA, AIA, $\mathrm{AG}_{3}$ y adenina (Tabla 1). El pH del medio de cultivo fue ajustado a 5,6. Se realizaron evaluaciones cada 7 días y la evaluación final se realizó 49 días después de la siembra.

Fase de multiplicación.- Para determinar el medio de cultivo más adecuado para la multiplicación de las plántulas, los tratamientos planteados consistieron en medio de cultivo base MS con diferentes concentraciones de BAP, KIN, ANA, AIA, $\mathrm{AG}_{3}$ y adenina (Tabla 2). Se realizaron evaluaciones cada 7 días y la evaluación final se realizó 35 días después de la siembra.

Fase de enraizamiento.- Para determinar el medio de cultivo adecuado para el enraizamiento de las plántulas se formularon diversos tratamientos empleando el medio base MS con diferentes concentraciones de IBA, BAP, ANA y adenina (Tabla 3). La evaluación se realizó 21 días después de la siembra.

Tabla 1. Medios de cultivo para la fase de establecimiento de meristemos.

\begin{tabular}{|c|c|}
\hline Tratamiento & Medio + Hormonas \\
\hline TRO & MS \\
\hline TR1 & $\mathrm{MS}+\mathrm{BAP} 0,5 \mathrm{mg} \cdot \mathrm{L}^{-1}+$ ANA $0,3 \mathrm{mg} \cdot \mathrm{L}^{-1}$ \\
\hline TR2 & $\mathrm{MS}+\mathrm{BAP} 0,5 \mathrm{mg} \cdot \mathrm{L}^{-1}+\mathrm{ANA} 0,3 \mathrm{mg} \cdot \mathrm{L}^{-1}+\mathrm{AG}_{3} 0,3 \mathrm{mg} \cdot \mathrm{L}^{-1}$ \\
\hline TR3 & $\mathrm{MS}+\mathrm{BAP} 0,5 \mathrm{mg} \cdot \mathrm{L}^{-1}+$ ANA $0,3 \mathrm{mg} \cdot \mathrm{L}^{-1}+$ Adenina $10 \mathrm{mg} \cdot \mathrm{L}^{-1}$ \\
\hline TR4 & $\mathrm{MS}+\mathrm{BAP} 0,5 \mathrm{mg} \cdot \mathrm{L}^{-1}+$ AIA $0,5 \mathrm{mg} \cdot \mathrm{L}^{-1}$ \\
\hline TR5 & $\mathrm{MS}+\mathrm{BAP} 0,5 \mathrm{mg} \cdot \mathrm{L}^{-1}+\mathrm{AIA} 0,5 \mathrm{mg} \cdot \mathrm{L}^{-1}+\mathrm{AG}_{3} 0,3 \mathrm{mg} \cdot \mathrm{L}^{-1}$ \\
\hline TR6 & $\mathrm{MS}+\mathrm{BAP}$ 0,5 mg. $\mathrm{L}^{-1}+$ AIA 0,5 mg. $\mathrm{L}^{-1}+$ Adenina $10 \mathrm{mg} \cdot \mathrm{L}^{-1}$ \\
\hline
\end{tabular}

Tabla 2. Medios de cultivo para la fase de multiplicación.

\begin{tabular}{|c|c|}
\hline Tratamiento & Medio + Hormonas \\
\hline TA 0 & MS \\
\hline TA 1 & $\mathrm{MS}+\mathrm{BAP} 0,5 \mathrm{mg} \cdot \mathrm{L}^{-1}+\mathrm{AIA} 0,3 \mathrm{mg} \cdot \mathrm{L}^{-1}+\mathrm{AG}_{3} 0,3 \mathrm{mg} \cdot \mathrm{L}^{-1}+$ Adenina $5 \mathrm{mg} \cdot \mathrm{L}^{-1}$ \\
\hline TA 2 & $\mathrm{MS}+\mathrm{BAP} 0,5 \mathrm{mg} \cdot \mathrm{L}^{-1}+\mathrm{AIA} 0,5 \mathrm{mg} \cdot \mathrm{L}^{-1}+\mathrm{AG}_{3} 0,3 \mathrm{mg} \cdot \mathrm{L}^{-1}$ \\
\hline TA 3 & $\mathrm{MS}+\mathrm{BAP} 0,5 \mathrm{mg} \cdot \mathrm{L}^{-1}+$ ANA $0,3 \mathrm{mg} \cdot \mathrm{L}^{-1}+\mathrm{AG}_{3} 0,3 \mathrm{mg} \cdot \mathrm{L}^{-1}+$ Adenina $5 \mathrm{mg} \cdot \mathrm{L}^{-1}$ \\
\hline TA 4 & $\mathrm{MS}+\mathrm{BAP} 0,5 \mathrm{mg} \cdot \mathrm{L}^{-1}+\mathrm{ANA} 0,5 \mathrm{mg} \cdot \mathrm{L}^{-1}+\mathrm{AG}_{3} 0,3 \mathrm{mg} \cdot \mathrm{L}^{-1}$ \\
\hline TA 5 & $\mathrm{MS}+\mathrm{KIN} 1 \mathrm{mg} \cdot \mathrm{L}^{-1}+$ AIA $0,3 \mathrm{mg} \cdot \mathrm{L}^{-1}+\mathrm{AG}_{3} 0,3 \mathrm{mg} \cdot \mathrm{L}^{-1}+$ Adenina $5 \mathrm{mg} \cdot \mathrm{L}^{-1}$ \\
\hline TA 6 & $\mathrm{MS}+\mathrm{KIN} 1 \mathrm{mg} \cdot \mathrm{L}^{-1}+\mathrm{AIA} 0,5 \mathrm{mg} \cdot \mathrm{L}^{-1}+\mathrm{AG}_{3} 0,3 \mathrm{mg} \cdot \mathrm{L}^{-1}$ \\
\hline TA 7 & $\mathrm{MS}+\mathrm{KIN} 1 \mathrm{mg} \cdot \mathrm{L}^{-1}+$ ANA $0,3 \mathrm{mg} \cdot \mathrm{L}^{-1}+\mathrm{AG}_{3} 0,3 \mathrm{mg} \cdot \mathrm{L}^{-1}+$ Adenina $5 \mathrm{mg} \cdot \mathrm{L}^{-1}$ \\
\hline TA 8 & $\mathrm{MS}+\mathrm{KIN} 1 \mathrm{mg} \cdot \mathrm{L}^{-1}+\mathrm{ANA} 0,5 \mathrm{mg} \cdot \mathrm{L}^{-1}+\mathrm{AG}_{3} 0,3 \mathrm{mg} \cdot \mathrm{L}^{-1}$ \\
\hline
\end{tabular}

Tabla 3. Medios de cultivo para la fase de enraizamiento.

\begin{tabular}{|c|c|}
\hline Tratamiento & Medio + Hormonas \\
\hline TM0 & MS \\
\hline TM1 & $\mathrm{MS}+\mathrm{IBA} 3 \mathrm{mg} \cdot \mathrm{L}^{-1}+$ ANA $0,5 \mathrm{mg} \cdot \mathrm{L}^{-1}$ \\
\hline TM2 & $\mathrm{MS}+\mathrm{ANA} 1 \mathrm{mg} \cdot \mathrm{L}^{-1}$ \\
\hline TM3 & $\mathrm{MS}+\mathrm{IBA} 3 \mathrm{mg} \cdot \mathrm{L}^{-1}$ \\
\hline TM4 & $\mathrm{MS}+$ IBA 3 mg. $\mathrm{L}^{-1}$ + Adenina 5 mg. $\mathrm{L}^{-1}$ \\
\hline TM5 & $\mathrm{MS}+\mathrm{IBA} 2 \mathrm{mg} \cdot \mathrm{L}^{-1}+\mathrm{BAP} 0,5 \mathrm{mg} \cdot \mathrm{L}^{-1}+\mathrm{AIA} 0,5 \mathrm{mg} \cdot \mathrm{L}^{-1}$ \\
\hline TM6 & $\mathrm{MS}+\mathrm{IBA} 2 \mathrm{mg} \cdot \mathrm{L}^{-1}+\mathrm{BAP} 0,5 \mathrm{mg} \cdot \mathrm{L}^{-1}+$ ANA $0,5 \mathrm{mg} \cdot \mathrm{L}^{-1}$ \\
\hline
\end{tabular}


Tabla 4. Influencia de los diferentes medios de cultivo en la diferenciación de meristemos apicales.

\begin{tabular}{cccc}
\hline \multirow{2}{*}{ Tratamientos } & \multicolumn{3}{c}{ 49 días } \\
\cline { 2 - 4 } & Meristemos diferenciados (\%) & Altura de plántulas (mm) & Número de brotes \\
\hline TR 0 & 87,5 & $2,3929 \mathrm{~d}$ & $1,0000 \mathrm{~d}$ \\
TR 1 & 87,5 & $7,6667 \mathrm{~b}$ & $1,1667 \mathrm{bcd}$ \\
TR 2 & 93,75 & $5,3000 \mathrm{c}$ & $1,0667 \mathrm{~cd}$ \\
TR 3 & 93,75 & $5,8000 \mathrm{c}$ & $1,1333 \mathrm{bcd}$ \\
TR 4 & 100 & $8,5313 \mathrm{ab}$ & $1,4375 \mathrm{~b}$ \\
TR 5 & 100 & $7,8750 \mathrm{ab}$ & $1,3750 \mathrm{bc}$ \\
TR 6 & $\mathbf{1 0 0}$ & $\mathbf{9 , 1 8 7 5 ~ a}$ & $\mathbf{2 , 1 8 7 5} \mathbf{a}$ \\
\hline
\end{tabular}

* Medias con diferentes letras en la misma columna difieren para p<0,05 (Notación Duncan)

\section{Resultados y discusión}

Fase de establecimiento.- En el 2002, Baca determinó que el explante más adecuado para el establecimiento in vitro de papaya eran los meristemos.

No se observó contaminación en la introducción in vitro de meristemos apicales de papaya. El uso de alcohol (70\%) durante 30 segundos y $\mathrm{NaOCl}(1 \%)$ durante 10 minutos resultó ser muy efectivo en la desinfección de explantes, pero es muy importante considerar que la introducción de meristemos se hizo a partir de plántulas provenientes de invernadero.

En la Tabla 4 se observa el porcentaje de meristemos diferenciados a los 49 días, las variaciones encontradas entre los distintos tratamientos se debieron básicamente a la manipulación de las yemas apicales durante la disección de los meristemos.

El análisis de los resultados a los 49 días después de la siembra nos indica que el tratamiento TR6 presenta mayor altura de plántula y mayor número de brotes (Tabla 4), estos datos nos indican que la combinación de los reguladores de crecimiento empleados en TR6 son óptimos para el establecimiento in vitro de meristemos apicales de papaya var. PTM-331.

Un balance apropiado entre auxinas y citoquininas en el medio de cultivo es necesario para la formación de plantas a partir de meristemos, ápices o yemas. Usualmente en los meristemos y ápices la citoquininas endógena es baja debido a que el principal sitio de síntesis son las raíces, por lo que la adición exógena de citoquininas en los medios de establecimiento es generalizada
(Jiménez 1998). El tratamiento TR6, el cual resultó ser el más óptimo para el establecimiento in vitro de meristemos apicales de la variedad de papaya PTM-331, contiene en su formulación 0,5 mg. $\mathrm{L}^{-1}$ de BAP, resultados congruentes a los obtenidos por Baca (2002) quien reporta una mayor proliferación de brotes de papaya empleando BAP como fuente de citoquininas.

Jiménez (1998) indica que los ápices y meristemos que son empleados como material inicial para el cultivo in vitro, son áreas de síntesis de auxinas por lo que la concentración endógena es alta y que normalmente cuando se emplean ápices no se adicionan auxinas al medio de cultivo aunque estos pueden estimular el crecimiento, pero cuando se emplean meristemos es frecuente que no exista suficiente auxina endógena siendo necesaria la adición exógena. Se puede observar que para el establecimiento in vitro de meristemos apicales de la variedad de papaya PTM-331, nuestra mejor fuente de auxinas fue el AIA a diferencia de los resultados obtenidos por Baca (2002) donde el ANA como fuente de auxinas resultó ser más efectivo para la diferenciación de meristemos de papaya criolla. En la tabla 4 se observa que los tratamientos que contienen ANA como fuente de auxinas (TR1, TR2 y TR3), presentaron porcentajes altos de diferenciación pero los explantes obtenidos presentaron menor altura de plántula y menor número de brotes que los explantes obtenidos con el tratamiento TR6. Alvarado (1992) indica que la utilización del AIA como fuente de auxina resulta más favorable que el ANA para el establecimiento in vitro de explantes de papaya.

Tabla 5. Altura de las plántulas $(\mathrm{mm})$ y número de brotes en la fase de multiplicación.

\begin{tabular}{|c|c|c|}
\hline \multirow[t]{2}{*}{ Tratamientos } & \multicolumn{2}{|c|}{35 días } \\
\hline & Altura de la plántula (mm) & Número de brotes \\
\hline TA0 & $5,5833 \mathrm{~g}$ & $1,0000 \mathrm{e}$ \\
\hline TA1 & $13,6667 \mathrm{~b}$ & $2,1667 \mathrm{~b}$ \\
\hline TA2 & 15,4167 a & 3,4167 a \\
\hline TA3 & $11,8333 \mathrm{~cd}$ & $2,0833 \mathrm{~b}$ \\
\hline TA4 & $11,3333 \mathrm{~d}$ & $2,0000 \mathrm{bc}$ \\
\hline TA5 & $13,7500 \mathrm{~b}$ & $2,0833 \mathrm{~b}$ \\
\hline TA6 & $12,1667 \mathrm{c}$ & $1,8333 \mathrm{bc}$ \\
\hline TA7 & 8,8333 e & $1,5833 \mathrm{~cd}$ \\
\hline TA8 & $7,1667 \mathrm{f}$ & 1,1667 de \\
\hline
\end{tabular}

${ }^{*}$ Medias con diferentes letras en la misma columna difieren para $p<0,05$ (Notación Duncan) 


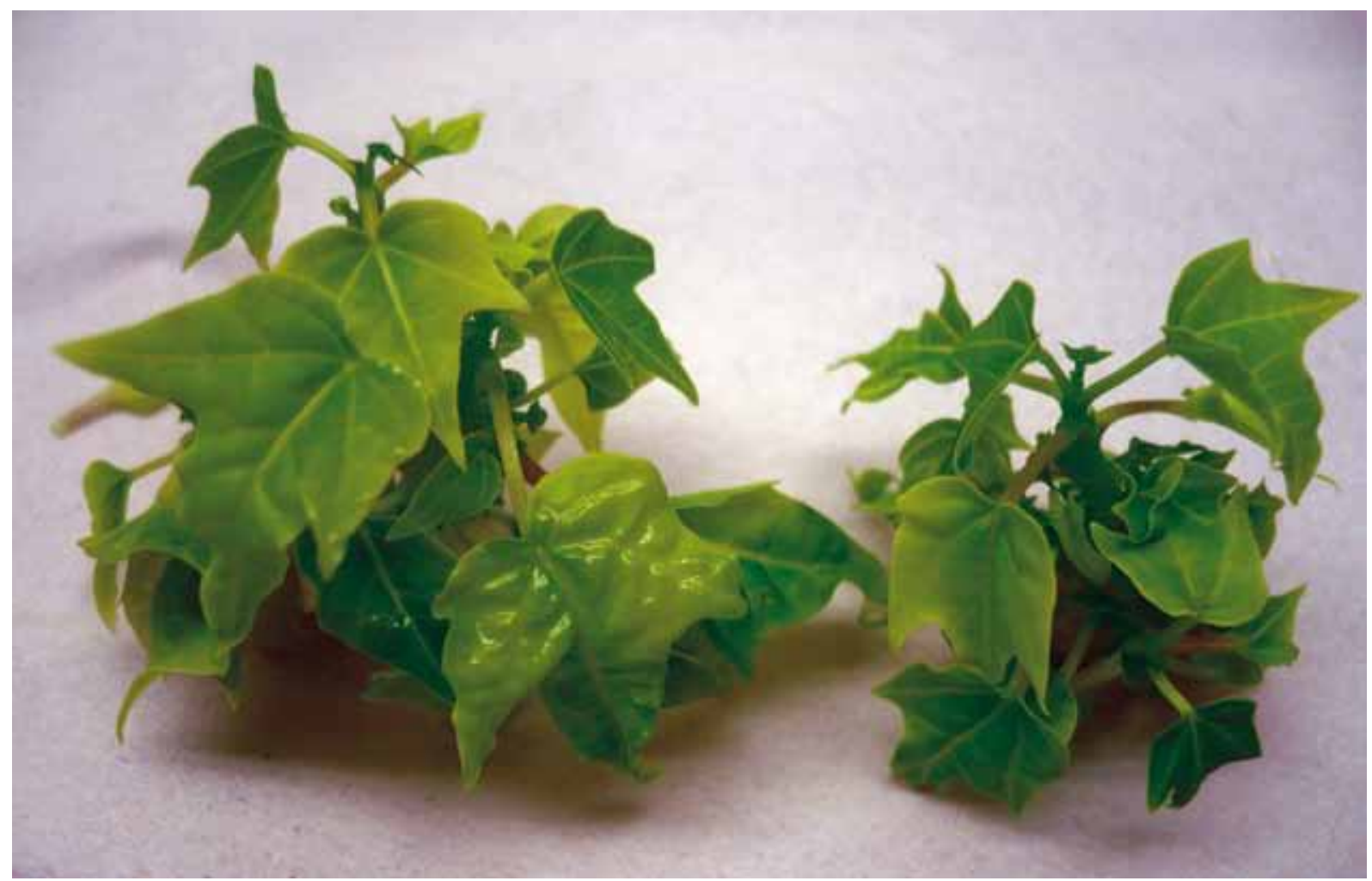

Figura 1. Plántulas provenientes de TA2, después de 35 días de siembra.

Fase de multiplicación.- El análisis de los resultados obtenidos al comparar el efecto de diferentes combinaciones de reguladores de crecimiento demostró que TA2 nos permitió obtener plántulas de mayor tamaño y con mayor número de brotes, con diferencias significativas frente al resto de los tratamientos (Tabla 5). La Figura 1 muestra las plántulas obtenidas en TA2. TA1 y TA5 también mostraron buenos resultados, sin diferencias significativas entre ellas, pero son significativamente diferentes al resto de tratamientos.

$\mathrm{Al}$ analizar la composición de los reguladores de crecimiento en TA1, TA2 y TA5 se observa que los tres tratamientos presentan AIA como fuente de auxina y estos son superiores a los tratamientos que presentan ANA como fuente de auxina.

En los tratamientos TA1 y TA2 la fuente de citoquininas es BAP y en el tratamiento TA5 la fuente de citoquininas es KIN, estos resultados nos indican que tanto BAP y KIN nos pueden proporcionar buenos resultados en la fase de multiplicación. Todos los tratamientos contienen $\mathrm{AG}_{3}$ en su composición debido a que esta hormona induce el alargamiento de los brotes. Baca (2002) obtuvo un mayor alargamiento de tallos empleando un medio basal MS al que se le adicionó $0,5 \mathrm{mg} \cdot \mathrm{L}^{-1} \mathrm{de}^{\mathrm{AG}_{3}}$ y 10 mg. $\mathrm{L}^{-1}$ de adenina.

El número de brotes es una variable importante en la fase de multiplicación ya que a mayor número de brotes se tendrá una mayor tasa de multiplicación. Orellana (1998) indica que la proliferación de brotes axilares se logra con la adición de citoquininas en el medio de cultivo para romper la dominancia apical y estimular la brotación de las yemas que se encuentran en las axilas de las hojas. El BAP resultó ser la citoquinina más efectiva para la multiplicación de brotes. Alvarado (1992) concluyó que la interacción de $0,05 \mathrm{mg} . \mathrm{L}^{-1}$ de AIA, 1,5 mg. $\mathrm{L}^{-1} \mathrm{de}$ KIN y 100 mg.L.-1 de ácido nicotínico permite la mayor proli- feración de nudos y desarrollo de abundante de follaje, mientras que Baca (2002) obtuvo mayor proliferación de brotes, mayor número de nudos y menor presencia de callos en un medio MS suplementado con $0,5 \mathrm{mg} \cdot \mathrm{L}^{-1}$ de BAP, $0,1 \mathrm{mg} \cdot \mathrm{L}^{-1}$ de $\mathrm{AG}_{3}, 0,01$ mg. $\mathrm{L}^{-1}$ de AIA y $10 \mathrm{mg} . \mathrm{L}^{-1}$ de adenina; en ambos casos el AIA fue la mejor fuente de auxinas.

Gallardo et al. (2002) reportaron que el medio de cultivo MS suplementado con 0,14 mg. $\mathrm{L}^{-1}$ de ANA y 0,45 mg. $\mathrm{L}^{-1}$ de BAP permite obtener mayores coeficientes de multiplicación.

La diferencia en la fuente de auxina más efectiva en la multiplicación in vitro se debe principalmente a la variedad de papaya empleada en cada caso, en el presente trabajo se empleó la variedad PTM-331; Alvarado (1992) empleó la variedad Pauna, Baca (2002) empleó la variedad Criollo y Gallardo et al. (2002) emplearon el híbrido IBP-4299.

Fase de enraizamiento.- El enraizamiento se caracteriza por ser la fase más voluminosa de todo el proceso, pues en ella cada brote, esqueje o yema de forma individual que se ha formado durante la fase de multiplicación, debe ser cultivada y manipulada in vitro para que, además de crecer y desarrollar un seudotallo o tallo con las primeras hojas, forme y desarrolle varias raíces que le permitan comenzar la absorción de nutrientes al transplantarse sobre un sustrato enriquecido y convertirse en vitroplanta aclimatizada lista para llevarse al campo (Orellana 1998).

Previamente al análisis estadístico de las variables número y longitud de raíces se determinó el porcentaje de enraizamiento en cada tratamiento. Se observó que TM0, TM2 y TM6 no presentaron enraizamiento por lo que estos tratamientos no fueron tomados en cuenta para el análisis estadístico. Se alcanzaron mejores resultados en TM4 con 83,33\% de plantas enraizadas (Tabla 6). 
Tabla 6. Número y longitud de raíces (21 días después de siembra).

\begin{tabular}{cccc}
\hline \multirow{2}{*}{ Tratamientos } & \multicolumn{3}{c}{ 21 días } \\
\cline { 2 - 4 } & Porcentaje de enraizamiento & Número de raíces & Longitud de raíces (mm) \\
\hline TM1 & $50 \%$ & $6,3333 \mathrm{~b}$ & $5,1667 \mathrm{~b}$ \\
TM3 & $66,67 \%$ & $8,5000 \mathrm{a}$ & $6,9375 \mathrm{a}$ \\
TM4 & $\mathbf{8 3 , 3 3 \%} \%$ & $\mathbf{8 , 6 0 0 0 \mathrm { a }}, 4000 \mathrm{a}$ & $4,1250 \mathrm{c}$ \\
\hline
\end{tabular}

* Medias con diferentes letras en la misma columna difieren para p<0,05 (Notación Duncan)

$\mathrm{Al}$ realizarse el análisis para las variables número y longitud de raíces, los tratamientos TM3 y TM4 presentan los mayores promedios, estos tratamientos no presentan diferencias significativas entre si de acuerdo a la prueba de Duncan realizada con un nivel de significancia de $\mathrm{p}<0,05$ (Tabla 6). Estas variables son de mucha importancia en la fase de aclimatación, debido a que es necesaria que una planta tenga el soporte de las raíces para un mejor desarrollo en el sustrato estéril. Si bien las raíces no son del todo funcionales debido a la escasez de pelos absorbentes que no se desarrollan in vitro, un mayor tamaño y un mayor número de raíces inducen la formación ex vitro de nuevas raíces y pelos absorbentes, que extraerán del suelo los nutrientes necesarios para el desarrollo de la plántula.

Al comparar los diferentes tratamientos, se observa que TM4 presenta el mayor porcentaje de enraizamiento, mayor número de raíces y mayor longitud de raíces; este tratamiento contiene el medio basal MS al que se le adicionó $3 \mathrm{mg} \cdot \mathrm{L}^{-1}$ de IBA y 5 mg. $\mathrm{L}^{-1}$ de adenina. Estos resultados se asemejan a los obtenidos por Baca (2002) que obtuvo mayor proliferación de raíces en un medio que contenía $2 \mathrm{mg} . \mathrm{L}^{-1}$ de IBA y $10 \mathrm{mg} . \mathrm{L}^{-1}$ de adenina.

En el tratamiento TM3 que contiene $3 \mathrm{mg} \mathrm{l}^{-1}$ IBA se observa un menor porcentaje de enraizamiento, pero las respuestas en el número de raíces y longitud de raíces no presentan diferencias significativas con las respuestas obtenidas en el tratamiento TM4. Reuveni et al. (1990) lograron enraizar plantas in vitro en un medio MS que contenía $1 \mathrm{mg} . \mathrm{L}^{-1}$ de IBA, mientras que Alvarado (1992) señala que el enraizamiento de plántulas de papaya se obtiene en un medio que contenga $5 \mathrm{mg} . \mathrm{L}^{-1} \mathrm{de}$ IBA.

Jiménez (1999), señala que el método de Drew (1993) induce el enraizamiento de los brotes en proliferación, es decir una concentración de $0,2 \mathrm{mg} . \mathrm{L}^{-1}$ de IBA y $11,3 \mathrm{mg} . \mathrm{L}^{-1}$ de Riboflavina. Posada (2005) indica que para el enraizamiento de plántulas de papaya, los explantes se subcultivan en un medio de cultivo compuesto por el $50 \%$ de las sales MS y concentraciones entre 3 y $5 \mathrm{mg} \cdot \mathrm{L}^{-1}$ de IBA durante 10 días, luego se transfieren a un medio de cultivo compuesto por sales MS sin reguladores de crecimiento por espacio de 20 días proporcionando un $80 \%$ de plantas enraizadas.

A manera de sumario puede señalarse que:

En la fase de establecimiento, el tratamiento donde se obtuvo una mayor diferenciación de meristemos a los 49 días fue TR6 (MS + 0,5 mg. $\mathrm{L}^{-1}$ de BAP + 0,5 mg. $\mathrm{L}^{-1}$ de AIA + $10 \mathrm{mg} \cdot \mathrm{L}^{-1} \mathrm{de}$ Adenina) y por lo tanto proporcionó plántulas más vigorosas para iniciar la fase de multiplicación.
El medio TA2 que contiene MS + 0,5 mg. $\mathrm{L}^{-1}$ de $\mathrm{BAP}+0,5$ mg. $L^{-1}$ de AIA + 0,3 mg. $L^{-1}$ de $A^{3}$, permitió obtener a los 35 días plántulas más grandes y con mayor número de brotes.

El uso de AIA como fuente de auxina nos permitió obtener mejores resultados que el uso del ANA en el establecimiento in vitro de meristemos y la multiplicación de explantes.

El tratamiento TM4 que posee MS + $3 \mathrm{mg} \cdot \mathrm{L}^{-1}$ de IBA + 5 mg. $\mathrm{L}^{-1}$ de adenina permitió obtener un mayor porcentaje de enraizamiento $(83,33 \%)$.

\section{Literatura citada}

Alvarado M. 1992. Propagación vegetativa in vitro del papayo (Carica papaya L.). Tesis Biólogo. Universidad Nacional Agraria La Molina. Lima, Perú. 60 pp.

Ascencio A., H. Gutiérrez, B. Rodríguez, et al. 2008. Plant regeneration of Carica papaya $\mathrm{L}$. through somatic embryogenesis in response to light quality, gelling agent and phloridzin. Scientia Horticulturae. 118 (2): 155-160.

Baca A. 2002. Optimización de la micropropagación in vitro de Carica papaya L. Tesis Ing. Agrónomo. Universidad Nacional Agraria La Molina. Lima, Perú. 107 pp.

Carbajal T. \& R. Remuzgo. 2007. Guía técnica del cultivo del papayo. Instituto de Investigaciones de la Amazonía Peruana. Programa de Biodiversidad. Tingo María, Perú. 40 pp.

Franciosi R. 1992. El cultivo del papayo en el Perú. Ediciones Fundeagro. Lima, Perú. 87 pp.

Gallardo J., L. Posada, R. Gómez, et al. 2002. Micropropagación del híbrido cubano de Papaya IBP 42-99. Biotecnología Vegetal. 2 (4): 211-215.

Ibar L. 1979. Aguacate, Chirimoyo, Mango y Papaya. Editorial Aedos. Barcelona, España. 173 pp.

Jiménez E. 1998. Cultivo de ápices y meristemos. En J. Pérez (Ed.), Propagación y mejora genética de plantas por biotecnología (pp. 45-56). Cuba. Ediciones GEO.

Jiménez C. 1999. Inducción de embriogénesis somática en papayo (Carica papaya Linnaeus) cultivar PT-101-B. Tesis Biólogo. Universidad Nacional Agraria La Molina. Lima, Perú. 82 pp.

Murashige T. \& F. Skoog. 1962. A revised medium for rapid growth and bioassays with tobacco tissue culture. Physiology plantarum. 15: 473-497.

Orellana P. 1998. Propagación vía organogénesis. En J. Pérez (Ed.), Propagación y mejora genética de plantas por biotecnología (pp. 151-178). Cuba. Ediciones GEO.

Posada L. 2005. Aplicaciones de la biotecnología a la propagación de la papaya. Biotecnología Vegetal. 5 (2): 67-79.

Reuveni D., D. Shlesinger \& U. Lavi. 1990. In vitro clonal propagation of dioecious Carica papaya L. Plant cell, tissue and organ culture. 20 (1): 41-46. 
